Pecvnia, Monográfico (2008), pp. 65-87

\title{
Cambio en la naturaleza del negocio de las entidades de crédito españolas en la segunda mitad de la década de los noventa (1995-2000): Evidencia empírica, nuevos problemas y soluciones organizativas
}

Este artículo examina el comportamiento de algunos parámetros económicos claves de las entidades de crédito españolas en el periodo 1995-2000. Dicho examen pone de manifiesto la continua disminución del margen de intermediación y sus causas, así como el cambio en la naturaleza de negocio bancario en el que cede importancia la actividad tradicional de intermediación adquiriendo mayor peso el negocio financiero (Intervención en los mercados de capitales, monetarios, de derivados, etc.). La extensión del ámbito de actividad bancaria da lugar a nuevas y especializadas unidades estratégicas de negocios que operan en condiciones de información asimétrica. Ello hace

\author{
Federico Marbella Sánchez \\ federico.marbella@unileon.es \\ Universidad de León \\ Organización de Empresas \\ Fac. de Ciencias Económicas y Empresariales \\ Campus de Vegazana, $\mathrm{s} / \mathrm{n}$ \\ 24071 León (España)
}

This paper examines the behaviour of several key economic parameters among Spanish finance companies over the period 1995-2000. This examination serves to highlight a continual decrease in margins for intermediaries and its causes. It also indicates the changes in the nature of banking business, in which the traditional function as intermediary is losing its prime place and financial business (intervention in capital, money, and derivatives markets and the like) is taking on a greater importance. This extension of the field of operations of the banking industry is giving rise to new specialized strategic business units operating in conditions 
necesario contar con diseños organizativos que faciliten la coordinación, reduzcan costes de estructura y de agencia y permitan explotar y/o generar sinergias entre las tradicionales $y$ las nuevas líneas de negocios.

Palabras clave: Entidades de crédito españolas, cambios en la naturaleza del negocio. of asymmetry of information. This leads to a need for organizational frameworks facilitating co-ordination, reducing agency costs and allowing synergies between traditional and new lines of business to be generated and-or exploited.

Key words: Spanish finance companies, changes in the nature of business.

\section{INTRODUCCIÓN}

El objetivo de este articulo es describir algunos de los cambios que han experimentado las entidades de crédito españolas (bancos, cajas y cooperativas de crédito) ${ }^{1}$ en la segunda mitad de década de los noventa, 1995-2000, en aspectos esenciales de su negocio, apuntar algunos de los nuevos problemas organizativos y las soluciones dadas en búsqueda de la eficiencia.

El modelo conceptual que sirve de hilo conductor es el que se recoge en la Figura 1 propuesto por Ballarín (1988) en el que partiendo de un esquema resumido de la cuenta de resultados de las entidades de crédito y de sus componentes se vincula el comportamiento de tales componentes al impacto que sobre los mismos ejercen determinadas fuerzas, denominadas "Palancas de gestión". Tales palancas se corresponden en gran medida con las fuerzas competitivas del esquema de Porter (1982).

El periodo elegido (1995-2000) cubre un espacio temporal suficiente para obtener una perspectiva aceptable y no es excesivamente amplio en un sector como el de las entidades de crédito en que los cambios se producen con gran celeridad y profundidad. A la vez este periodo coincide con un ciclo de bonanza económica y sin crisis importantes de las entidades de crédito, lo que permite observar una evolución no perturbada por fenómenos distintos de los del propio comportamiento del negocio financiero.

1 Las empresas, objeto de análisis son el colectivo de bancos, cajas y cooperativas de crédito, que denominaremos "entidades de crédito" para distinguirlas de otros establecimientos financieros de crédito ( $\mathrm{S}$. de Crédito hipotecario, S. de Arrendamiento financiero, de Financiación, de Factoring). 
Figura 1: Palancas de gestión en el sector de servicios financieros

\begin{tabular}{|c|c|}
\hline Cuenta de resultados & Palancas de gestión \\
\hline $\begin{array}{l}\text { Ingresos financieros } \\
\text { - Gastos financieros } \\
\text { Margen financiero } \\
\text { + Ingresos por servicios } \\
\text { Margen bruto } \\
\text { - Costes de transformación } \\
\text { Fondos generados } \\
\text { - Amortización y provisiones } \\
\text { Beneficio bruto } \\
\text { - Impuestos } \\
\text { Beneficio neto } \\
\text { - Dividendos } \\
\text { Beneficio retenido }\end{array}$ & $\begin{array}{l}\text { - Poder de negociación con los demandantes de fondos } \\
\text { - Poder de negociación con los proveedores de fondos } \\
\text { - Gestión del riesgo de intereses } \\
\text { Copacidad de innovación } \\
\text { Coordinación } \\
\text { - Gestión de riesgo de insolvencia }\end{array}$ \\
\hline
\end{tabular}

Fuente: Adaptado de Ballarín (1988).

Los aspectos que se analizarán para evidenciar el cambio son: el estrechamiento del margen financiero, la diversificación de las fuentes de ingresos, el esfuerzo en la contención de costes de transformación y la mejora de la solidez y solvencia financiera de tales entidades. Se ha optado por el análisis de estos aspectos por la relevancia para la configuración y dirección del negocio de tales entidades tratando de obtener una visión de conjunto lo más integrada posible de una realidad extremadamente amplia y compleja ${ }^{2}$.

La información cuantitativa utilizada procede básicamente de los boletines estadísticos y económicos del Banco de España, que se nutren de la procedente de las cuentas y estados financieros que las entidades de crédito proporcionan a sus respectivos Organismos o Instituciones supervisoras y se contrasta los resultados en algunos aspectos con otras investigaciones.

2 Una amplia revisión del estado actual de la investigación en temas de Dirección Estratégica Bancaria puede verse en Sanchís (2001). 


\section{EVIDENCIA EMPÍRICA DEL CAMBIO}

Estrechamiento del margen financiero o de intermediación. Uno de los elementos fundamentales para captar la evolución del núcleo central del negocio de las entidades de crédito es el comportamiento del margen financiero o de intermediación, determinado como diferencia entre los productos financieros obtenidos por los intereses de las operaciones de activo y los gastos financieros derivados de las operaciones de pasivo.

El spread o diferencial (margen de intermediación) depende de múltiples factores, entre los que se encuentra la estructura del mercado en el que actúan los intermediarios financieros (Carrasco y Domínguez 1995). Habitualmente tiende a considerarse una relación de signo negativo entre un mercado de competencia y los márgenes financieros, asociación lógica bajo los postulados económicos estándares y que se confirman con la caída de los mismos en las entidades de crédito españolas ante una mayor competencia ${ }^{3}$.

Tabla 1: Margen de intermediación (\% s/ATM) en las entidades de crédito españolas

\begin{tabular}{lccccccc}
\hline & $\mathbf{1 9 9 5}$ & $\mathbf{1 9 9 6}$ & $\mathbf{1 9 9 7}$ & $\mathbf{1 9 9 8}$ & $\mathbf{1 9 9 9}$ & $\mathbf{2 0 0 0}$ & $\begin{array}{c}\text { Variación \% } \\
\mathbf{2 0 0 0 / 1 9 9 5}\end{array}$ \\
\hline Bancos & 2,28 & 2,05 & 1,96 & 1,88 & 1,80 & 1,81 & $-20,61$ \\
\hline Cajas de ahorro & 3,49 & 4,7 & 3,19 & 2,94 & 2,76 & 2,66 & $-23,79$ \\
\hline Cooperativas de crédito & 4,51 & 4,234 & 3,98 & 3,7 & 3,51 & 3,41 & $-24,39$ \\
\hline Total entidades de crédito & 2,78 & 2,54 & 2,44 & 2,30 & 2,20 & 2,18 & $-21,83$ \\
\hline
\end{tabular}

Fuente: Banco de España. Boletines económicos, abril 1999, pp. 92-95, y abril 2001, pp. 122-125. Elaboración propia.

En el periodo 1995-2000 se ha producido una disminución del margen de intermediación del $21,83 \%$ en el conjunto de los tres colectivos que compiten para satisfacer las necesidades de demandantes y depositantes de fondos. La mayor caída se da en las cooperativas de crédito pese a lo cual su ratio mantiene la mejor posición relativa a finales del año 2000. La ventaja en margen de las cooperativas de crédito denota un mayor poder de negociación de las mismas sobre proveedores y demandantes de fondos potenciado a raíz de la ley de cooperativas de crédito de 1989 que las dota de una mayor libertad para operar con otro tipo de clientes distintos de los cooperativistas (Sanchís y Camps 2003).

3 Un análisis comparativo de la evolución de los márgenes y de la rentabilidad del sistema bancario en los países de la OCDE se encuentra en Carrasco y Domínguez (1995) Papeles de Economía Española, 65, pp. 160-173. 
A pesar de que el margen de intermediación de los bancos a finales del siglo XX es inferior al de los otros dos grupos de competidores son precisamente los bancos los que actualmente están soportando mejor la presión sobre dicho margen. Este hecho es relevante porque se trunca la tendencia que según Martín (1997) y Fernández y Suárez (1998) era habitual y que consistía en que las cajas de ahorro sufrían menores reducciones de sus márgenes que los propios bancos.

Un primer análisis para comprender el comportamiento del margen de intermediación implica comparar la evolución de la rentabilidad de la inversión crediticia y el coste de pasivo. La rentabilidad crediticia ha sufrido un profundo recorte que se eleva hasta un de $47,94 \%$. Las fuerzas del mercado han determinado caídas similares en los tres colectivos (bancos, cajas de ahorro y cooperativas de crédito) hasta el punto de casi igualar la rentabilidad crediticia de las mismas a finales del año 2000 convirtiendo a los tres colectivos en alternativas con similar grado de atractivo para satisfacer las necesidades de los demandantes de fondos (Tabla 2).

Tabla 2: Rentabilidad crediticia (\%) en las entidades de crédito españolas

\begin{tabular}{lccccccc}
\hline & $\mathbf{1 9 9 5}$ & $\mathbf{1 9 9 6}$ & $\mathbf{1 9 9 7}$ & $\mathbf{1 9 9 8}$ & $\mathbf{1 9 9 9}$ & $\mathbf{2 0 0 0}$ & $\begin{array}{c}\text { Variación \% } \\
\mathbf{2 0 0 0} / \mathbf{1 9 9 5}\end{array}$ \\
\hline Bancos & 10,95 & 10,09 & 7,93 & 6,52 & 5,21 & 5,70 & $-47,94$ \\
\hline Cajas de Ahorro & 10,83 & 10,40 & 8,43 & 6,82 & 5,45 & 5,65 & $-47,83$ \\
\hline Cooperativas de crédito & 11,63 & 11,07 & 8,89 & 7,14 & 5,83 & 5,91 & $-49,18$ \\
\hline Total entidades de depósito & 10,93 & 10,25 & 8,18 & 6,67 & 5,34 & 5,69 & $-47,94$ \\
\hline
\end{tabular}

Fuente: Banco de España. Boletines económicos, abril 1999, p. 99, y abril 2001, p. 129. Elaboración propia.

La reducción de la rentabilidad de la inversión crediticia, está relacionada con el aumento de poder de negociación de los demandantes de fondos, una de las fuerzas o palancas de gestión del modelo que sirve de hilo conductor del trabajo (Figura 1). Tal poder se ha elevado debido a la mayor cultura e información financiera de los demandantes de fondos, a la posibilidad de estos de conectarse directamente con los ahorradores y a la ampliación de las alternativas para obtener fondos y prestación de servicios de otras instituciones tales como compañías de seguros, sociedades de crédito hipotecario y bancos de inversión e incluso de empresas no financieras en el proceso de modernización de las finanzas españolas (Castelló 1996). 
Una de las respuestas a la reducción de la rentabilidad crediticia, como no podía menos ser, ha sido la reducción de la retribución del pasivo (depósitos), con el propósito de mantener el margen de intermediación (Tabla 3).

Tabla 3: Coste medio del total de los pasivos financieros onerosos (\%)

\begin{tabular}{lccccccc}
\hline & $\mathbf{1 9 9 5}$ & $\mathbf{1 9 9 6}$ & $\mathbf{1 9 9 7}$ & $\mathbf{1 9 9 8}$ & $\mathbf{1 9 9 9}$ & $\mathbf{2 0 0 0}$ & $\begin{array}{c}\text { Variación \% } \\
\mathbf{2 0 0 0 / 1 9 9 5}\end{array}$ \\
\hline Bancos & 7,53 & 7,02 & 5,13 & 4,15 & 3,31 & 4,08 & $-45,82$ \\
\hline Cajas de ahorro & 6,23 & 5,48 & 4,23 & 3,28 & 2,25 & 2,78 & $-55,38$ \\
\hline Cooperativas de crédito & 5,87 & 5,50 & 3,76 & 2,79 & 1,78 & 2,20 & $-62,52$ \\
\hline Total entidades de depósito & 7,06 & 6,58 & 4,79 & 3,81 & 2,88 & 3,54 & $-49,86$ \\
\hline
\end{tabular}

Fuente: Banco de España. Boletines económicos, abril 1999, p. 99, y abril 2001, p. 129. Elaboración propia.

La reducción del coste medio del pasivo (depósitos) ha sido de un $49,86 \%$ para el conjunto de los tres colectivos, ligeramente superior a la caída de la rentabilidad crediticia $(47,94 \%)$, lo que lleva a explicar la caída del margen de intermediación más por volumen (proceso de desintermediación) que en términos de precios y costes unitarios (tipos de interés de activo y pasivo). En efecto, precio y coste del dinero evolucionan de forma bastante paralela (Figura 2) y en todo caso con reducción ligeramente mayor de los costes del pasivo que de los rendimientos del activo que no se han ajustado en su totalidad al nuevo coste medio, especialmente en operaciones que tienen fijado un "stop floor" o suelo.

Figura 2: Evolución de rentabilidad crediticia y coste del pasivo

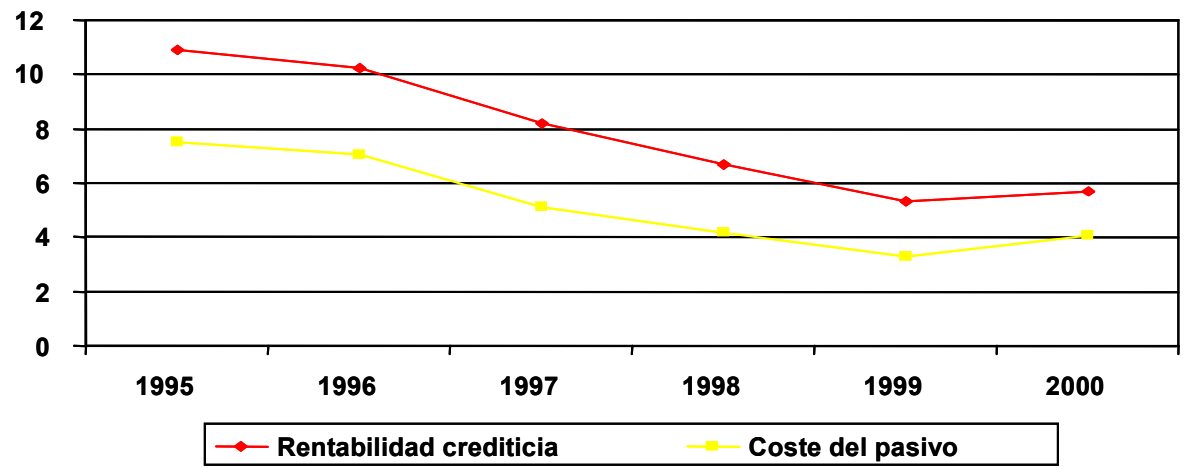

La reducción del margen de intermediación y su explicación por más volumen que por precio concuerda con el mayor grado de 
madure $z^{4}$ que van alcanzado el sector que conlleva a que éste opere con tasas de crecimiento cada vez más moderadas. En efecto, los activos medios de los tres colectivos en conjunto crecieron en el periodo 1995-2000 a una tasa media anual acumulativa del $9 \%$, pero ya en los últimos años 2000 y 1999 se reduce el ritmo a tasas mas bajas en torno al 6\% (Banco de España. Boletines Económicos, abril 1999, abril 2001).

Detrás de la reducción del margen de intermediación subyacen además factores como la progresiva apertura del mercado doméstico potenciada por el ingreso de nuestro país en la Unión Económica y Monetaria (UEM) ${ }^{5}$ y la irrupción de los nuevos canales de distribución (banca por Internet, banca telefónica, etc.). Estos hechos irrefutables reducen las barreras de entrada, de naturaleza legal y económica, reducción que ha sido más fuerte a partir de la adopción del Acta Única (1985) y de la utilización intensiva de las nuevas tecnologías de la información (T.I.).

Otra de las palancas que afecta al comportamiento del margen financiero y que se puede utilizar para mejorar su posición es la Gestión del riesgo de intereses. La utilidad de este instrumento de análisis y gestión deriva de su capacidad para poner de manifiesto cómo el hecho de que las inversiones crediticias tengan vencimientos distintos a los de los pasivos dar lugar a rendimientos diferentes en función de la estructura temporal de los tipos de interés, con el consiguiente impacto en el margen financiero ${ }^{6}$.

Búsqueda de nuevas fuentes de ingresos. La caída del margen de intermediación ha forzado la búsqueda de nuevos ingresos y la paulatina transición hacia el cobro de comisiones por los servicios asociados a la utilización de los sistemas de pagos y por operaciones financieras distintas de las tradicionales de intermediación financiera (Martín 1997). El cambio de política supone una transición hacia un modelo en que cada usuario paga por los productos y servicios que utiliza, eliminándose las

\footnotetext{
El grado de madurez de un sector puede ser medido por la tasa de crecimiento de variables relevantes o claves del sector (cifra de ventas, volumen de negocio, inversión en activos, etc.).

5 Un resumen de las etapas y actuaciones más importantes llevadas a cabo en el camino hacia la moneda única se halla en Sebastián (1999).

6 Una exposición de las técnicas de Gestión del riesgo de intereses puede verse en Casilda, Lamothe y Monjas (1997).
} 
subvenciones cruzadas implícitas que la práctica anterior comportaba (Basurto 2001).

La orientación hacia el cobro de los servicios en los años analizados es un hecho que ha continuado durante los años más recientes. En efecto, como se aprecia en la Tabla 4 las comisiones netas percibidas por las entidades de crédito han pasado de representar un $0,52 \%$ sobre los activos totales medios en 1995 a un $0,69 \%$ en el 2000 con un incremento del 32,69\%. El mayor crecimiento relativo de las comisiones de los bancos se debe, según Fernández y Suárez (1998), a la mayor variedad de servicios que ofrecen a su clientela, entre las que destacan las empresas a las que atienden en mayor medida los bancos; según Sanchís y Camps (2003) los menores ingresos por servicios en las cooperativas de crédito serían reflejo de la menor capacidad de innovación.

Tabla 4: Comisiones netas percibidas (\%s/ATM's)

\begin{tabular}{lccccccc}
\hline & $\mathbf{1 9 9 5}$ & $\mathbf{1 9 9 6}$ & $\mathbf{1 9 9 7}$ & $\mathbf{1 9 9 8}$ & $\mathbf{1 9 9 9}$ & $\mathbf{2 0 0 0}$ & $\begin{array}{c}\text { Variación \% } \\
\mathbf{2 0 0 0} / \mathbf{1 9 9 5}\end{array}$ \\
\hline Bancos & 0,55 & 0,54 & 0,62 & 0,68 & 0,72 & 0,76 & 38,18 \\
\hline Cajas de ahorro & 0,48 & 0,50 & 0,58 & 0,64 & 0,63 & 0,61 & 27,08 \\
\hline Cooperativas de crédito & 0,44 & 0,46 & 0,50 & 0,59 & 0,60 & 0,60 & 36,36 \\
\hline Total entidades de depósito & 0,52 & 0,52 & 0,60 & 0,66 & 0,68 & 0,69 & 32,69 \\
\hline
\end{tabular}

Fuente: Banco de España. Boletines económicos, abril 1999, pp. 92-95 y abril 2001, pp. 122-125. Elaboración propia.

El incremento de ingresos por servicios financieros se ha visto impulsado por la introducción de innovaciones que Rodríguez (1997) clasifica en innovaciones financieras puras e innovaciones tecnológicofinancieras ${ }^{7}$. La innovación es otra de las palancas de gestión señaladas en el modelo que constituye el hilo conductor del análisis.

Asimismo el crecimiento de la contratación de valores de renta fija, variable, la comercialización de nuevos productos financieros como planes y fondos de inversión y toma de participaciones en compañías financieras y no financieras ha generado un aumento de esta línea de negocio (comisiones) que van modificando la estructura de la cuenta de resultados y del negocio bancario.

7 Por Innovaciones financieras puras entiende Rodríguez (1997) aquellas que aun apoyándose en un cierto nivel de tecnología apenas guardan relación con la misma y por innovaciones tecnológico-financieras aquellas que tienen su origen o se apoyan básicamente en las nuevas tecnologías. 
En cuanto a la toma de participaciones en compañías financieras y no financieras, dos tipos de factores han potenciado las tomas de participaciones en empresas no financieras: a) Factores de tipo externo como la liberalización del sector de servicios, especialmente telecomunicaciones (telefonía móvil, cable, radio, televisión) y la posterior privatización parcial de las grandes empresas del sector público español (telecomunicaciones, energía y eléctricas), b) Factores internos como el estrechamiento de márgenes financieros de las entidades de crédito lo que las está obligando a buscar inversiones de mayor rentabilidad (Sanchís, Puig y Soriano 1998).

La inversión en la cartera de valores de renta variable ${ }^{8}$, incluidas todo tipo de participaciones (en empresas financieras, instrumentales y no financieras) ha aumentado en el conjunto de las entidades de crédito en el periodo $1995-2000$ un 76\% (Tabla 5). El mayor crecimiento se produce en las cajas de ahorro con incremento sobre sus activos medios del $125 \%^{9}$ representando a finales del año 2000 un $5,4 \%$ de tales activos; en las cooperativas de crédito el aumento ha sido del $80 \%$, a pesar de lo cual su cartera de valores aún tiene escaso peso, un 1,8\% sobre los activos totales medios; en los bancos con un crecimiento del $65 \%$ la cartera de renta variable tiene mayor ponderación representando un $6,6 \%$ de los activos medios ${ }^{10}$.

Un análisis empírico efectuado por Sanchís, Puig y Soriano (1998) para una muestra de 18 entidades de crédito (ocho bancos y diez cajas) que representan más del $50 \%$ del volumen de activos, referido a participaciones en empresas no financieras en el periodo 1990-1997, apunta tendencias similares a las que se derivan de la información general del Banco de España, es decir, de crecimiento y de mayor intensidad en las cajas de ahorro que en los bancos.

8 La cartera de renta variable considerada incluye tanto la inversión en activos financieros rentables en pesetas como en moneda extranjera; esta última con menor ponderación.

9 Una de las cajas que ha seguido una clara estrategia de toma de participaciones en empresas industriales ha sido la Caixa que se ha convertido en accionista relevante en compañías notables como Repsol, Gas Natural o Telefónica.

10 La crisis de los años 70 puso de manifiesto la debilidad de los bancos industriales como bancos especializados en la financiación a medio y largo plazo de las empresas no financieras, desapareciendo algunos y/o reconvirtiéndose otros. Por ello en un proceso de sustitución paralelo los antiguos grandes bancos amplían sus participaciones en empresas y sectores relevantes o considerados estratégicos por los mismos (Banco Bilbao en el sector alimenticio y eléctrico, Banco Central y Banesto en el sector energético, Banco Hispano Americano en el de la construcción). 
Cambio en la naturaleza del negocio de las entidades de crédito españolas en la segunda mitad de la década de los noventa (1995-2000)

Tabla 5: Evolución de la cartera de Renta variable (\%s/ATM's)

\begin{tabular}{lccccccc}
\hline & $\mathbf{1 9 9 5}$ & $\mathbf{1 9 9 6}$ & $\mathbf{1 9 9 7}$ & $\mathbf{1 9 9 8}$ & $\mathbf{1 9 9 9}$ & $\mathbf{2 0 0 0}$ & $\begin{array}{c}\text { Variación \% } \\
\mathbf{2 0 0 0 / 1 9 9 5}\end{array}$ \\
\hline Bancos & 4,0 & 4,1 & 4,1 & 4,1 & 4,9 & 6,6 & 65 \\
\hline Cajas de ahorro & 2,4 & 2,9 & 3,6 & 4,2 & 4,5 & 5,4 & 125 \\
\hline Cooperativas de crédito & 1,0 & 1,1 & 1,4 & 1,7 & 1,8 & 1,8 & 80 \\
\hline Total entidades de depósito & 3,4 & 3,5 & 3,8 & 4,1 & 4,6 & 6,0 & 76 \\
\hline
\end{tabular}

Fuente: Banco de España. Boletines económicos, abril 1999, p. 100 y abril 2001, p. 130. Elaboración propia.

El incremento de la cartera de valores conlleva implicaciones que Sanchís y Soriano (2001) clasifican en económicas (rendimientos por dividendos, de ampliaciones de capital, de emisiones de obligaciones convertibles, de venta de opciones, etc.) por su posesión como inversión y en estratégicas y demás derechos políticos asociados a la parte proporcional de capital que se posee. Las actividades de diversificación posicionándose en sectores tan importantes como las telecomunicaciones y energía es un factor más que modifica la naturaleza del negocio de las entidades de crédito.

La mayor ponderación de la cartera de renta variable en los bancos se justifica por la tradicional relación entre banca privada y empresas no financieras que se inicia con la creación de la banca industrial, de negocios e inversiones y se consolida con la constitución en su día de grandes grupos bancarios como BBV, BCH, Santander, Banesto (Sanchís y Soriano 2001). El crecimiento de la participación en empresas de la economía real no es una característica exclusiva de las entidades de crédito españolas sino que sigue la orientación general que se viene dando en otros países y en los del área euro (Basurto 2001).

Los cambios descritos, junto con la mayor presencia de las entidades de crédito en los mercados financieros ${ }^{11}$, como respuesta al fenómeno de la desintermediación, están provocando un cambio naturaleza del negocio bancario: En efecto, una reciente estimación realizada por la Asociación Española de Banca en base a criterios de diferenciación de líneas de negocio y de atribución de costes constata dicho cambio (Tabla 6).

11 La desintermediación financiera tanto desde la óptica de los depositantes (proveedores de fondos) como desde la óptica de los prestatarios (tomadores de fondos), la entrada de nuevos competidores bancarios y no bancarios, la legislación favorable de la UE y el deseo de no perder cuota ha forzado a las entidades financieras a prestar servicios más completos y sofisticados incluyendo la presencia creciente en mercados de capitales. 
Tabla 6: Evolución de las líneas del negocio bancario (Adoptado de Basurto 2001)

\begin{tabular}{lcccc}
\hline & \multicolumn{2}{c}{$\mathbf{1 9 9 2 - 1 9 9 3}$} & \multicolumn{2}{c}{$\mathbf{1 9 9 9 - 2 0 0 0}$} \\
\hline & $\begin{array}{c}\text { Negocio } \\
\text { tradicional }\end{array}$ & $\begin{array}{c}\text { Negocio } \\
\text { financiero }\end{array}$ & $\begin{array}{c}\text { Negocio } \\
\text { tradicional }\end{array}$ & $\begin{array}{c}\text { Negocio } \\
\text { financiero }\end{array}$ \\
\hline Atribución de Activos Totales Medios (\%) & 78,9 & 21,1 & 79,1 & 20,9 \\
\hline Contribución al beneficio neto (\%) & 84,4 & 15,6 & 42,5 & 57,5 \\
\hline Fuente: AEB (Asociación Española de Banca). & & & &
\end{tabular}

Según tal estimación en 1992-1993 una aplicación del 78,9\% de los activos al negocio tradicional aportaba un $84,4 \%$ al beneficio neto y el $21,1 \%$ de los atribuidos al nuevo negocio financiero aportaba el $15,6 \%$ restante al beneficio; en 1999-2000 una aplicación del 79,1\% al negocio tradicional sólo contribuye al beneficio en un $42,5 \%$ y el $20,9 \%$ aplicado al financiero llega a aportar hasta un $57,5 \%$. Es claro que se están produciendo cambios en la naturaleza del negocio bancario en el que la actividad tradicional (intermediación) pierde peso frente a las de mayor componente financiero (mercados de capitales, carteras de valores, fondos de inversión, etc.) que se está revelando según tal estimación como de mayor eficiencia en la relación beneficio/activos empleados.

La búsqueda de nuevas fuentes de ingresos ha forzado también el desarrollo de una estrategia de expansión geográfica, de internacionalización de la actividad bancaria. El proceso de internacionalización de la banca ha pasado por diferentes etapas que Durán (2002) sintetiza en las siguientes: a) Hasta 1986 (incorporación de España a la (EE) la banca seguía a las empresas y a los trabajadores españoles en sus actividades en el exterior, b) 1986-1992 (Integración Europea I, integración de España en la (EE), se sigue la orientación al Mercado Interior Europeo, c) 1992-1998, se orienta hacia la multinacionalización en América Latina y alianzas estratégicas y cooperación en Europa. d) 1998-Actual (Integración Europea II, Unión Monetaria, Euro) se persigue la consolidación y expansión en Ibero América y el crecimiento en Europa. En el proceso de internacionalización destaca la de los grandes bancos en la década de los noventa y comienzos del nuevo siglo dirigida fundamentalmente a Ibero América (Brasil, Argentina, México, etc.).

Contención de costes. El estrechamiento del margen de intermediación exigía una respuesta de las entidades de crédito en términos de contención de costes de transformación o gastos de explotación (Tabla 7). 
Tabla 7: Gastos de explotación (\%s/ATM's)

\begin{tabular}{lccccccc}
\hline & $\mathbf{1 9 9 5}$ & $\mathbf{1 9 9 6}$ & $\mathbf{1 9 9 7}$ & $\mathbf{1 9 9 8}$ & $\mathbf{1 9 9 9}$ & $\mathbf{2 0 0 0}$ & $\begin{array}{c}\text { Variación \% } \\
\mathbf{2 0 0 0 / 1 9 9 5}\end{array}$ \\
\hline Bancos & 2,01 & 1,91 & 1,85 & 1,78 & 1,75 & 1,70 & $-15,42$ \\
\hline Cajas de ahorro & 2,61 & 2,50 & 2,48 & 2,40 & 2,27 & 2,15 & $-17,62$ \\
\hline Cooperativas de crédito & 2,90 & 2,75 & 2,76 & 2,69 & 2,56 & 2,43 & $-16,21$ \\
\hline Total entidades de depósito & 2,23 & 2,13 & 2,09 & 2,01 & 1,96 & 1,89 & $-15,25$ \\
\hline
\end{tabular}

Fuente: Banco de España. Boletines económicos, abril 1999, pp. 92-95, y abril 2001, pp. 122-125. Elaboración propia.

La reducción de los gastos de explotación o transformación sobre activos medios en el conjunto de los tres colectivos (bancos, cajas y cooperativas de crédito) ha sido importante, del 15,25\% en el periodo considerado. Se ha efectuado básicamente sobre los gastos de personal, propiciada por los procesos de concentración bancaria ${ }^{12}$, por la introducción de las nuevas tecnologías, por el incremento del outsourcing tanto en servicios centrales como oficinas, por jubilaciones anticipadas y la contratación a bajo coste de un mayor número de jóvenes titulados que puede atender una mayor variedad y complejidad de trabajos (Bueno y Borrajo 2001).

Tabla 8: Ratio de eficiencia (Gastos de explotación/Ingresos básicos)

\begin{tabular}{lccccccc}
\hline & $\mathbf{1 9 9 5}$ & $\mathbf{1 9 9 6}$ & $\mathbf{1 9 9 7}$ & $\mathbf{1 9 9 8}$ & $\mathbf{1 9 9 9}$ & $\mathbf{2 0 0 0}$ & $\begin{array}{c}\text { Variación \% } \\
\mathbf{2 0 0 0 / 1 9 9 5}\end{array}$ \\
\hline Bancos & 0,70 & 0,71 & 0,69 & 0,67 & 0,67 & 0,63 & $-10,00$ \\
\hline Cajas & 0,65 & 0,65 & 0,65 & 0,66 & 0,66 & 0,65 & 0,00 \\
\hline Cooperativas de crédito & 0,59 & 0,58 & 0,61 & 0,62 & 0,62 & 0,60 & $+1,69$ \\
\hline Total entidades de depósito & 0,68 & 0,68 & 0,67 & 0,66 & 0,66 & 0,64 & $-5,88$ \\
\hline
\end{tabular}

Fuente: Banco de España. Boletines económicos, abril 1999, pp. 92-95, y abril 2001, pp. 122-125. Elaboración propia.

El esfuerzo de contención de costes ha llevado a la mejora del ratio de eficiencia ${ }^{13}$; en los bancos pasa de un 0,70 en 1995 a un 0,63 en el 2000 , en las cajas de ahorro y cooperativas de crédito, con ligeras oscilaciones, se mantiene prácticamente igual al final del periodo que al

12 El sector bancario español ha vivido durante los años más recientes un importante proceso de concentración, a través de las fusiones de las entidades más grandes, que como es lógico se ha visto reflejado en los indicadores que pueden utilizarse para medir el mencionado fenómeno.

13 El ratio de eficiencia se calcula por la relación entre los gastos de explotación e ingresos básicos, entendidos estos últimos como los financieros netos y los obtenidos por comisiones. 
comienzo del mismo. El mayor peso relativo de los bancos hace que la evolución de este importante ratio en el conjunto de las entidades de crédito sea favorable (Tabla 8 ). Los avances en materia de eficiencia en los bancos son importantes si bien aún no alcanzan los ratios teóricos considerados como satisfactorios que se sitúan en torno al 50\% (Bueno y Borrajo 2001).

Solidez y solvencia de las entidades de crédito. La solidez y solvencia ligada a la palanca "Gestión del riesgo de insolvencia" depende en gran medida del ciclo económico o de la mala suerte, en terminología anglosajona efecto bad luck (Bueno y Borrajo 2001) y tiene repercusión en las políticas de provisiones reflejadas en la cuenta de resultados resumida (Figura 1 ).

El periodo que se analiza se caracteriza por una evolución positiva del ciclo económico y de estabilidad. Ello ha llevado a un fortalecimiento de la calidad de las carteras de inversión de las entidades de crédito, tanto por la disminución de las tasas de morosidad, como por el incremento de su cobertura propiciado por la existencia de beneficios altos. En efecto, la tasa de créditos dudosos / riesgo total \%, del conjunto de las entidades de crédito ha bajado de un $4,8 \%$ en 1995 a un $1 \%$ en el 2000 (Tabla 9) y el índice de saneamiento, relación (Provisiones/Dudosos \%) se sitúa muy claramente por encima del $100 \%$, en las cajas en un $176,8 \%$, en los bancos en un 168,8\% y en las cooperativas de crédito en un $133,0 \%$ (Tabla 10).

Tabla 9: Solidez y Solvencia (Créditos dudosos s/riesgo total \%)

\begin{tabular}{lccccccc}
\hline & $\mathbf{1 9 9 5}$ & $\mathbf{1 9 9 6}$ & $\mathbf{1 9 9 7}$ & $\mathbf{1 9 9 8}$ & $\mathbf{1 9 9 9}$ & $\mathbf{2 0 0 0}$ & $\begin{array}{c}\text { Variación \% } \\
\mathbf{2 0 0 0 / 1 9 9 5}\end{array}$ \\
\hline Bancos & 4,4 & 3,0 & 1,9 & 1,3 & 1,0 & 0,9 & $-79,54$ \\
\hline Cajas de ahorro & 5,4 & 4,2 & 2,8 & 1,8 & 1,2 & 1,0 & $-81,48$ \\
\hline Cooperativas de crédito & 5,3 & 4,2 & 3,1 & 2,5 & 1,8 & 1,6 & $-69,81$ \\
\hline Total entidades de depósito & 4,8 & 3,5 & 2,3 & 1,5 & 1,1 & 1,0 & $-79,17$ \\
\hline
\end{tabular}

Fuente: Banco de España. Boletines económicos, abril 1999, p. 110, y abril 2001, p. 140. Elaboración propia.

Tabla 10: Solidez y Solvencia (Provisiones /Créditos dudosos \%)

\begin{tabular}{lrrrrrrc}
\hline & $\mathbf{1 9 9 5}$ & $\mathbf{1 9 9 6}$ & $\mathbf{1 9 9 7}$ & $\mathbf{1 9 9 8}$ & $\mathbf{1 9 9 9}$ & $\mathbf{2 0 0 0}$ & $\begin{array}{c}\text { Variación \% } \\
\mathbf{2 0 0 0 / 1 9 9 5}\end{array}$ \\
\hline Bancos & 78,4 & 91,7 & 112,4 & 134,0 & 149,5 & 168,8 & 115,3 \\
\hline Cajas de ahorro & 70,1 & 78,8 & 96,9 & 117,6 & 145,0 & 176,8 & 152,2 \\
\hline Cooperativas de crédito & 82,3 & 91,5 & 106,6 & 115,4 & 115,3 & 133,0 & 61,60 \\
\hline Total entidades de depósito & 75,2 & 86,1 & 105,0 & 125,3 & 145,2 & 168,5 & 124,06 \\
\hline
\end{tabular}

Fuente: Banco de España. Boletines económicos, abril 1999, p. 110, y abril 2001, p. 140. Elaboración propia. 
También hay que señalar que el nivel relativo de recursos propios está por encima de los mínimos exigidos por los Acuerdos de Capital de Basilea $^{14}$, que buscaron homogeneidad de normativas nacionales y siguiendo tales orientaciones la Unión Europea exige desde el 1 de enero de 1993 un requerimiento básico de recursos propios del 8\% de los activos medios ponderados ${ }^{15}$.

La política de dividendos, última de las palancas de gestión considerada del modelo conceptual (Figura 1) está en gran medida determinada por la distinta naturaleza de las sociedades de los tres colectivos. Los bancos son sociedades anónimas con capital privado con amplia libertad para fijar la política de dividendos; las cajas de ahorro pueden ser definidas como instituciones sin capital social sustituido por el fondo fundacional simbólico al que no retribuyen, sólo con carácter residual deben aportar fondos a la obra social (el dividendo social); las cooperativas de crédito son empresas societarias cuyo capital está formado por las aportaciones de los cooperativistas en las que la distribución de beneficios esta regulada. Como no podía ser de otro modo, con unas bases conceptuales y legales distintas la posición cuantitativa de distribución dividendos y de retención de beneficios es también distinta (Tabla 11).

Tabla 11: Retención de beneficios (\%)

\begin{tabular}{lrrrrrr}
\hline & $\mathbf{1 9 9 5}$ & $\mathbf{1 9 9 6}$ & $\mathbf{1 9 9 7}$ & $\mathbf{1 9 9 8}$ & $\mathbf{1 9 9 9}$ & $\mathbf{2 0 0 0}$ \\
\hline Bancos & 31,4 & 36,3 & 31,9 & 36,0 & 28,5 & 32,7 \\
\hline Cajas de ahorro & $\mathbf{7 5 , 3}$ & 74,1 & 74,4 & 73,6 & 74,0 & 73,8 \\
\hline Cooperativas de crédito & $\mathbf{7 8 , 8}$ & 80,0 & 79,5 & 80,0 & 80,0 & 79,7 \\
\hline
\end{tabular}

Fuente: Banco de España. Boletines económicos, abril 1999, p. 110, y abril 2001, p. 140. Elaboración propia.

Se observa claramente un hecho y es que en las entidades en que la distribución de los resultados está regulada (cooperativas de crédito) o en las que no existe compromiso de retribución al capital por inexistencia del mismo (cajas de ahorro) la retención es muy superior a la de aquellas otras (los bancos) en las cuales los órganos de decisión gozan

\footnotetext{
14 Una reciente estimación de la Asociación Española de Banca sitúa el exceso de recursos propios sobre los mínimos exigidos en un $20 \%$.

15 La forma de cálculo y de detalle de las ponderaciones de los distintos tipos de activos puede verse en Casilda, Lamothe y Monjas (1997).
} 
de un mayor grado de discrecionalidad para llevar a cabo una política activa de reparto de beneficios.

\section{NUEVOS PROBLEMAS Y SOLUCIONES ORGANIZATIVAS}

El análisis efectuado pone de manifiesto un cambio en la naturaleza del negocio bancario con desplazamiento desde el negocio tradicional de intermediación hacia nuevos ámbitos de producto/mercado/ clientes (fondos de inversión, planes y fondos de pensiones, mercado de valores mobiliarios, participación en empresas, mercados hipotecarios, mercados geográficos distintos), cambio forzado por la mayor competencia y la necesidad de paliar la caída del margen de intermediación. La extensión del campo de actividad lleva a una concepción de banca universal en la que coexisten bajo un mismo centro de decisión distintas actividades o áreas de negocio (banca comercial, banca de empresas, banca de seguros, de mercado de capitales, banca privada, banca minorista, mayorista, de área de negocio de América, Europa, etc.), aumentan los tipos de producto, los segmentos atendidos y los sistemas de información y organización clásicos se ven desbordados. En síntesis, la universalidad aumenta la complejidad. Ello genera problemas de coordinación y agencia que hace necesario contar con soluciones organizativas eficientes.

Desde la Economía de las Organizaciones una entidad de crédito de carácter universal puede considerarse como un nexo de contratos con dos tipos de vínculos: El primero refleja los vínculos entre los trabajadores de la entidad y de las demás sociedades jurídicas en las que aquellos prestan sus servicios profesionales, el segundo los vínculos legales o puramente organizativas entre las unidades estratégicas de negocios (UEN) (comercial, de banca de empresas, banca privada, etc.) y de estas con el centro corporativo (Canals 1996).

Bajo la hipótesis de existencia de relaciones entre las unidades de negocios y el centro corporativo de la entidad de crédito de carácter universal, surgen problemas de agencia entre los directivos (principal) del centro y los de las distintas unidades estratégicas de negocios (agentes) debido a la existencia de información asimétrica. En efecto, los directivos de las distintas unidades estratégicas de negocios actúan en mercados especializados en algunos de los cuales además se toman decisiones rápidas, frecuentemente intradía (mercados bursátiles, interbancarios, de opciones, derivados, divisas, etc.) por lo que disponen 
y actúan en condiciones de información asimétrica lo que conduce al problema de la selección adversa (adverse selection) $)^{16}$.

Por otra parte la existencia de objetivos diferentes entre directivos del centro corporativo del banco universal (principal) y los de las unidades de negocios (agente) en la medida que los primeros no pueden controlar las acciones de los segundos, puede conducir a que estos persigan más sus propios intereses que los de la organización lo que conduce al problema del riesgo moral. Problema que se acrecienta por la habitual estructura asimétrica de los ingresos de los directivos de las UEN y los rendimientos obtenidos, que se concreta en que los directivos de las UEN de los bancos universales poseen un incentivo positivo (prima) si logran beneficios en sus transacciones pero no sufren detracción en el caso de que dichas transacciones generen resultados negativos. En consecuencia, no es el agente quién sufre los resultados negativos de sus actuaciones sino que las ineficiencias y costes se trasladan al banco universal a través de la UEN correspondiente. Las posibles soluciones que penalicen la retribución del agente pueden resultar invalidadas si el agente carece de capital suficiente para pagar las multas o perdidas que cause (Milgrom y Robert 1993).

En síntesis, la disposición de información asimétrica en poder de los directivos de las unidades estratégicas de negocios especializadas de los bancos universales puede conducir a comportamientos oportunistas de los mismos ya que toman decisiones que sólo ex post pueden ser valoradas por el centro corporativo, cuando los resultados de las decisiones son irreversibles ${ }^{17}$. Esta situación responde a la conocida como de acción no observable e información no observable "hidden action y hidden information" (Arrow 1985).

La solución a los problemas de la relación de agencia en la entidad con vocación de banca universal conlleva costes de diseñar contratos explícitos entre los directivos de las unidades estratégicas especializadas y el centro corporativo, costes de seguimiento, verificación y $\operatorname{control}^{18}$. A ello se añade que los contratos por ser internos tienen escasa validez

\footnotetext{
16 Una exposición de la teoría de la agencia y de los costes de agencia puede verse entre otros, en los trabajos de Jensen y Meckling (1976) y Ricart (1987).

17 Una exposición de concepto riesgo moral y sus implicaciones en los comportamientos y eficiencia de las transacciones se halla en Milgrom y Roberts (1993).

18 Un análisis más amplio a nivel general se encuentra en Arruñada (1990: 67-73).
} 
jurídica, sólo la relacionada con el establecimiento de sistemas de objetivos, métodos de evaluación de rendimientos y sistemas de remuneración que se aplican desde el centro corporativo a las unidades especializadas de negocios (Canals 1996).

A los problemas derivados de la información asimétrica (riesgo moral, selección adversa) de los bancos universales se añaden problemas de diseño organizativo para la coordinación de las distintas unidades de negocios, de motivación de los directivos del mismo nivel pero situados en unidades de negocios o divisiones con características competitivas distintas (banca comercial, de banca de inversiones, banca de empresas, etc.) y de reducir los costes de influencia. En organizaciones complejas, como es el caso de los bancos universales, los costes de influencia se manifiestan en aquellas actividades o decisiones que buscan trasladar costes o ingresos de unas unidades de negocios a otras (Canals 1996).

La solución a los nuevos problemas organizativos en las entidades de crédito de carácter universal requiere la implantación de diseños y mecanismos capaces: a) de minimizar los costes de coordinación y motivación, b) de eliminar o suavizar los problemas derivados de la información asimétrica (riesgo moral, selección adversa) y de las actividades de influencia. Solución nada sencilla pues como señala Fernández (1994) al referirse al problema del diseño organizativo no existen modelos únicos.

Una primera solución posible es el diseño organizativo divisional que desagrega la entidad de crédito en unidades de negocios denominadas divisiones ${ }^{19}$, a cuyo frente se sitúan los respectivos equipos, dependiendo de un directivo relevante (Director General o Consejero Delegado) del grupo bancario. El centro corporativo del banco se reserva las tareas básicas (dirección global de la corporación, coordinación de divisiones, organización del grupo, asignación de recursos, evaluación de rendimientos y provisión de servicios centrales, etc.) dotando mayores o menores grados de autonomía para el resto de las actividades o funciones a la UEN bancarias. La principal ventaja de esta forma organizativa es que permite pasar de la complejidad de la combinación de negocios bancarios a una consideración y tratamiento individualizado (Canals 1996). Por el

19 La estructura divisional nace a principios del siglo XX en el ámbito de las grandes empresas norteamericanas. Sobre esta forma organizativa puede verse Mintzberg (1988). 
contrario presenta como inconvenientes la necesidad de asignar activos y recursos previamente compartidos, el aislamiento al que se ve sometida cada división, la necesidad de establecer precios internos de transferencia por los servicios prestados entre las distintas divisiones o entre estas y el centro corporativo.

Otra solución es el modelo de banca federada desarrollado por el grupo Mckinsey en torno a una estructura tipo "Holding". En este modelo el banco se desagrega en unidades de negocio que constituyen empresas económica y jurídicamente independientes bajo el control de la empresa Holding. La desagregación en este modelo está orientada a alcanzar la diferenciación, las unidades se especializan unas en producción, otras en distribución y otras en procesamiento operando como negocios independientes, se centralizan las actividades susceptibles de generar sinergias y se contempla la posibilidad de subcontratar con terceros introduciendo la disciplina del mercado (Camps y Sanchís 2003) ${ }^{20}$.

La justificación para mantener en un banco universal UENs con alto grado de independencia y personalidad jurídica distinta radica en la gran especialización de algunos servicios (mercados de futuros, de derivados, divisas, etc.) que solo pueden prestarse eficientemente con un alto grado de autonomía, a la vez que así los problemas del riesgo moral del seguro de depósitos quedan limitados a las unidades que desarrollan la actividad bancaria tradicional y no afectarían a otros negocios de naturaleza más especulativos, heterogéneos o de mayor riesgo.

Una tercera posible solución es el esquema organizativo estructurado en forma de Trebol (Bueno 1992) diseñado para servir especialmente al modelo de banca virtual. La banca virtual es una nueva forma de concebir los servicios financieros en que no se necesitan emplazamientos físicos, las oficinas pierden importancia, permite la colaboración con los competidores, proveedores, clientes, la comunicación es telefónica o a través de maquinas y supone el dominio de las técnicas telefónicas y coordinación de los inputs de servicios (Castelló 1996). Este modelo no es sino una mezcla de tendencias estratégicas sin concreción estructural alguna, limitándose a proponer una organización bancaria que utilice intensamente el outsourcing, el down-sizing y los recursos

20 Un análisis comparativo entre modelo de banca federada y banca divisional se halla en el trabajo de Z. Fernández (1993) "La banca federada. Aportaciones y limitaciones de un nuevo modelo organizativo", Perspectivas del sistema financiero, 42, pp. 24-32. 
tecnológicos que permitan un ahorro de costes mediante el autoservicio bancario, recetas más que manidas por los profesionales del sector (Camps y Sanchís 2003).

En el caso de las entidades de crédito españolas como señalan Hernángomez et al. (1992), Bueno (1992), Azofra (1994), Castelló (1996), Fernández y Suárez (1998) se partía (década de los sesenta) de un contexto o "statu quo" poco diversificado y estable que llevó a definir su estructura organizativa primaria como una estructura funcional, de naturaleza burocrática maquinal y asimétrica debido a la primacía de las unidades de distribución (tupida red de oficinas) sobre cualquier otra.

Pues bien, ante los fuertes cambios en la estructura del negocio expuestos, sorprendentemente las entidades de crédito españolas han considerado demasiado arriesgado un cambio organizacional de tipo radical, realizando ajustes o adecuaciones graduales que no rompan de manera brusca con viejas organizaciones funcionales de naturaleza centralizada y trabajo burocrático maquinal (Camps y Sanchís 2003). En efecto, la reacción más generalizada ha consistido en mantener las estructuras primarias de naturaleza funcional con pequeños cambios vía descentralización, flexibilización, dotación de nuevas capacidades y aplanamiento jerárquico que permiten redefinirlas como estructuras funcionales simétricas con tendencias burocráticas profesionales muy puntuales ${ }^{21}$. Las excepciones quedan limitadas a un número reducido de casos entre los cuales como más conocidos se hallan el Caso Argentaria, Caso de las cajas de ahorro vascas BBK, grupo de cajas rurales, en que se han implantado diseños más avanzados que se aproximan al modelo de banca federada.

\section{CONCLUSIONES}

Se ha constatado un estrechamiento del margen financiero en los tres colectivos (bancos, cajas y cooperativas de crédito) que en conjunto en el periodo $1995-2000$ se ha reducido en un $21,83 \%$ sobre activos totales medios. Dicha reducción está relacionada con el aumento de poder de negociación los demandantes y proveedores de fondos que

21 En Camps y Sanchís (2003) puede verse el caso del Banco Popular cuyo modelo organizativo ha introducido cambios sobre la estructura primaria funcional. 
exigen mejores condiciones en precio y servicios, con la entrada al sector de nuevos competidores (banca extranjera, instituciones parafinancieras y no financieras), con la sucesiva eliminación de las barreras de entrada, fundamentalmente de naturaleza legal, con el ingreso de nuestro país en la UEM, con la irrupción de nuevos canales de distribución, la profundización en los procesos de desintermediación financiera y la reducción de tipos de interés marcada por Banco Central Europeo (BCE) y la Reserva Federal.

El estrechamiento del margen financiero ha forzado comportamientos estratégicos orientados a la búsqueda y diversificación de nuevas fuentes de ingresos, a través de cobro de comisiones por servicios, a la toma de posiciones y participaciones relevantes en renta variable en empresas industriales lo que supone una vuelta al modelo de banca universal, a una estrategia de expansión geográfica básicamente hacia países de Ibero América que incluye adquisiciones de entidades financieras autóctonas, a una estrategia deliberada de reducción de los costes de transformación y políticas tendentes al reforzamiento de la solidez y solvencia financiera de dichas entidades.

Los cambios producidos conllevan una transformación importante de la concepción y naturaleza del negocio bancario en el que pierde relevancia el negocio tradicional (intermediación) en favor del negocio financiero de mayor especialización. Ello hace surgir nuevas unidades estratégicas de negocio bancario que operan en condiciones de información asimétrica dando lugar a nuevos problemas de agencia y coordinación que deben ser solventados con modelos organizativos ad hoc que den soluciones eficientes. Las nuevas formulas organizativas adoptadas por las entidades de crédito españolas no ha supuesto una ruptura con las tradicionales. La reacción más generalizada ha consistido en mantener estructuras de naturaleza funcional con mayor grado de descentralización, flexibilización, dotación de nuevas capacidades y mayor aplanamiento jerárquico.

\section{BIBLIOGRAFÍA}

ARROW, K. (1985) "The Economics of Agency". J.W. PratT \& R.J. ZeCKHAUSER (Eds.) Principals and Agents: The Structure of Business. Boston: Harvard Business School Press. 
ARRUÑADA, B. (1990) Economía de la Empresa: Un enfoque contractual. Barcelona: Ariel.

ASOCIACIÓN ESPAÑOLA DE CONTABILIDAD Y ADMINISTRACIÓN DE EMPRESAS (AECA) (1991) Criterios de medición de la rentabilidad, productividad y eficiencia de las entidades bancarias, "Documentos", 5, Madrid.

AzofrA, V. (1994) "Estructura organizativa y resultados de las cajas de ahorro españolas", Papeles de Economía Española, 58, pp. 285-296.

BALLARín, E. (1989) Estrategias competitivas para la Banca, $2^{a}$ edición. Barcelona: Ariel.

- (1988) "Distribución de servicios financieros en España: Implicaciones competitivas", Papeles de Economía Española, 2, pp. 145-156.

BANCO DE ESPAÑA (1999/2001) Boletines económicos, abril 1999 y abril 2001.

BASURTO, J. (2001) "Nuevos Bancos para un Nuevo Siglo", Economistas, 89, pp. 16-22.

BRYAN, L.L. (1992) Bankrupt. New York: Harper Collins.

BuENO, E. (1992) "El cambio en la banca y las nuevas estructuras organizativas", Revista Española de Financiación y Contabilidad, 73, pp. 781-806.

- y F. BORRAJO (2001) "La evolución de la banca española en una década". E. BUENO y I. DE LA TORRE (Eds.) Evolución y Perspectivas de la Banca Española. Madrid: Civitas, pp. 75-100.

- y I. DE LA TORRE (2001) Evolución y Perspectivas de la Banca Española. Madrid: Civitas.

CAMPS, J. y J.R. SANCHÍS (2003) "Estudio del modelo organizativo federal. Análisis de su aplicación en las entidades de crédito españolas", Revista Europea de Dirección y Economía y de la Empresa, Vol. 12, 2, pp. 173200.

CANALS, J. (1996) Bancos universales y diversificación empresarial. Madrid: Alianza Editorial.

CARRAsCo, G. y J.M. Domínguez (1995) "Evolución de los márgenes y de la rentabilidad del sistema bancario en los países de la OCDE", Papeles de Economía Española, 65, pp. 160-173.

CASILDA, R.; P. LAMOTHE y M. MONJAS (1997) La banca y los mercados financieros. Madrid: Alianza Editorial.

CASTELLó, E. (1996) Dirección y Organización de Entidades financieras. Madrid: ESIC. 
CHULIÁ, C. (1990) "Las participaciones del sistema bancario en las empresas no financieras", Papeles de Economía Española, 44, pp. 73-87.

DURÁN, J.J. (2002) "La expansión multinacional de la banca española. Santander Central Hispano, S.A. (SCH), y Banco Bilbao Vizcaya Argentaria S.A. (BBVA)". M. García Olalla y C. VÁzQuez Ordás (eds.) Estrategias y Operaciones Empresariales en los Nuevos Mercados, 20 Casos a estudio. Madrid: Civitas, pp. 91-141.

FERNÁNDEZ, Z. (1994) "La banca universal: un futuro en entredicho", Papeles de Economía Española, 58, pp. 262-275.

- y I. SUÁREZ (1998) "Las cajas de ahorro: Estrategia y Estructura", Papeles de Economía Española, 74-75, pp. 202-221.

Goold, M. y A. CAMPBeLl (1989) Estrategias y Estilos. Madrid: Tecnologías de Gerencia y Producción.

-, - y AleXAnder, M. (1994) Corporate Level Strategy. Creating Value in the Multibusiness Company. New York: John Wiley \& Sons.

GUAL, J. (1994) La racionalización del sector bancario español. Bilbao: Fundación BBV.

Hernángomez, J.; V. AZOFra, J.M. de LA Fuente y J. GutiéRreZ (1992) La estructura organizativa de las cajas de ahorro, Confederación Española de Cajas de Ahorro.

Jensen, M.C. \& W.H. MECKLING (1976) "Theory of the Firm: Managerial Behavior, Agency Costs, and Ownership Structure", Journal of Financial Economics, 3, pp. 305-360.

MARTín, M. (1997) "Competitividad y eficiencia de las entidades de depósito españolas", Perspectivas del Sistema Financiero, 59, pp. 57-71.

MILGROM, P. y RoBerTS, J. (1993) Economía, Organización y Gestión de la Empresa. Barcelona: Ariel Economía.

MINTZBERG, H. (1988) La estructuración de las organizaciones. Barcelona: Ariel Economía.

PORTER, M. (1982) Estrategia competitiva. México: CECSA.

RICART, J.E. (1987) "Una introducción a los modelos de agencia", Revista Española de Economía, Vol. 4, 1, pp. 43-62.

SANCHÍs PALACIO, J.R. (2001) "Dirección Estratégica Bancaria: estado actual y temas de investigación", Cuadernos de Economía y de Dirección de la Empresa, 8, pp. 77-107.

- y J. CAMPS (2003) Dirección estratégica bancaria. Madrid: Díaz de Santos. 
- y J.F. SORIANO (2001) "Relaciones banca-Industria en España. Participaciones bancarias en el período 1992-1997", Economía Industrial, 341, pp. 55-66.

-, F. PUIG y J.F. SORIANO (1998) "Relaciones Banca-Industria. Un estudio empírico sobre las participaciones de entidades de crédito en el capital de las empresas no financieras", Perspectivas del Sistema Financiero, 63-64, pp. 83-102.

SEBASTIÁN, A. (1999) Nuevas estrategias del sistema bancario en la Unión Económica y Monetaria. Madrid: Instituto de Estudios Económicos. 